\title{
High resolution magnetic field measurements in high-mass star-forming regions using masers
}

\author{
Gabriele Surcis ${ }^{1}$, Wouter H. T. Vlemmings ${ }^{2}$, \\ Huib J. van Langevelde ${ }^{1,3}$ and Busaba Hutawarakorn Kramer ${ }^{4}$ \\ ${ }^{1}$ Joint Institute for VLBI in Europe \\ Postbus 2, 7990AA, Dwingeloo, the Netherlands \\ email: surcis@jive.nl \\ ${ }^{2}$ Chalmers University of Technology, Onsala Space Observatory \\ SE-439 92 Onsala, Sweden \\ email: wouter.vlemmings@chalmers.se \\ ${ }^{3}$ Sterrewacht Leiden, Leiden University \\ Postbus 9513, 2300RA Leiden, the Netherlands \\ email: langevelde@jive.nl \\ ${ }^{4}$ Max-Planck Institut für Radioastronomie \\ Auf dem Hügel 69, 53121 Bonn, Germany \\ email: bkramer@mpifr-bonn.mpg.de
}

\begin{abstract}
The bright and narrow spectral line emission of masers is ideal for measuring the Zeeman-splitting as well as for determining the orientation of magnetic fields in 3-dimensions around massive protostars. Recently, polarization observations at milliarcsecond resolution of 6.7- $\mathrm{GHz} \mathrm{CH}_{3} \mathrm{OH}$ masers have uniquely been able to resolve the morphology of magnetic fields close to massive protostars. The observations reveal that the magnetic fields are along outflows and/or on the surfaces of circumstellar tori. Here we present three different examples selected from a total number of 7 massive star-forming regions that were investigated at $6.7-\mathrm{GHz}$ with the EVN in the last years.
\end{abstract}

Keywords. Stars:formation, masers:methanol, polarization

\section{Introduction}

Three different scenarios have been proposed to explain the formation of high-mass stars. In one of these scenarios, Core accretion (McKee \& Tan 2003), massive stars form through gravitational collapse, which involves disc-assisted accretion to overcome radiation pressure. This scenario is similar to the favored picture of low-mass star-formation, in which magnetic fields are thought to play an important role by removing excess angular momentum, thereby allowing accretion to continue onto the star. However, the role of magnetic fields during the protostellar phase of high-mass star-formation is still a debated topic. In particular, it is still unclear how magnetic fields influence the formation and dynamics of discs and outflows. Most current information on magnetic fields close to high-mass protostars comes from polarized maser emissions, which allow us to investigate the magnetic field on small scales (10s-1000s AU) by using interferometers, such as the European VLBI Network (EVN) and the Very Long Baseline Array (VLBA).

In this contribution we summarize the results obtained by observing the full polarized emission of $6.7-\mathrm{GHz}_{\mathrm{CH}} \mathrm{OH}$ towards the massive protostars W75N-VLA 1, NGC7538IRS 1, and W51-e2. For W75N-VLA 1 and NGC7538-IRS 1 we also observed the polarized emission of $22-\mathrm{GHz} \mathrm{H}_{2} \mathrm{O}$ masers. The linear polarization and total intensity of 


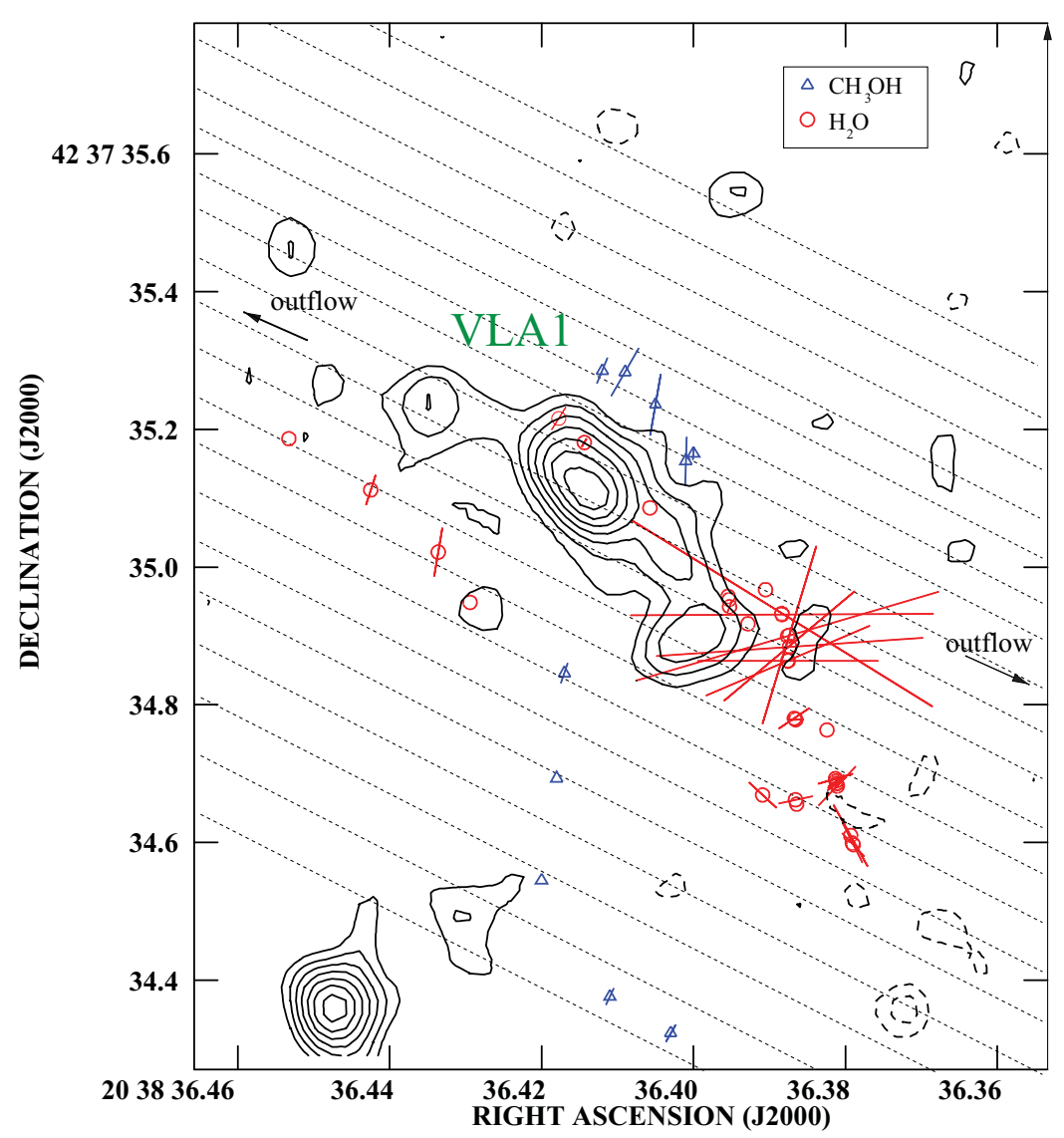

Figure 1. Modified version of Fig.3 of Surcis et al. (2011b) of the $\mathrm{CH}_{3} \mathrm{OH}$ (triangles) and $\mathrm{H}_{2} \mathrm{O}$ (circles) masers of W75N-VLA 1. The contours are the $1.3 \mathrm{~cm}$ continuum emission observed with the VLA. The linear polarization vectors are also reported (20 mas correspond to a linear polarization fraction of $1 \%$ ). The dashed lines indicate the large-scale direction of the magnetic field.

22- $\mathrm{GHz} \mathrm{H}_{2} \mathrm{O}$ and 6.7-GHz $\mathrm{CH}_{3} \mathrm{OH}$ masers were analysed by using a full radiative transfer method code based on the models of Nedoluha \& Watson (1992). The output of the code are the emerging brightness temperature $\left(T_{\mathrm{b}} \Delta \Omega\right)$ and the intrinsic thermal linewidth $\left(\Delta V_{\mathrm{i}}\right)$ from which the $\theta$ angle between the magnetic field orientation and the maser propagation direction can be estimated (e.g., Surcis et al. 2011a). From the circularly polarized emission of the masers we were able to measure the Zeeman-splitting of the two maser transitions, but only in the case of $\mathrm{H}_{2} \mathrm{O}$ masers this led to the magnetic field strength, as the value of the Landé $g$-factor appropriate for the $6.7-\mathrm{GHz} \mathrm{CH}_{3} \mathrm{OH}$ masers is still unknown (Vlemmings et al. 2011).

\section{W75N-VLA 1}

VLA 1 is a massive protostar located in the active high-mass star-forming region $\mathrm{W} 75 \mathrm{~N}(\mathrm{~B})$ (Torrelles et al. 1997), which is at a distance of $1.30 \pm 0.07 \mathrm{kpc}$ (Rygl et al. 2012). In the region two compact H II regions were also detected, named VLA 2 and VLA 3, which are though to be in an earlier evolutionary stage than VLA 1 (Torrelles et al. 1997). A large-scale high-velocity outflow, with an extension greater than $3 \mathrm{pc}$ and 


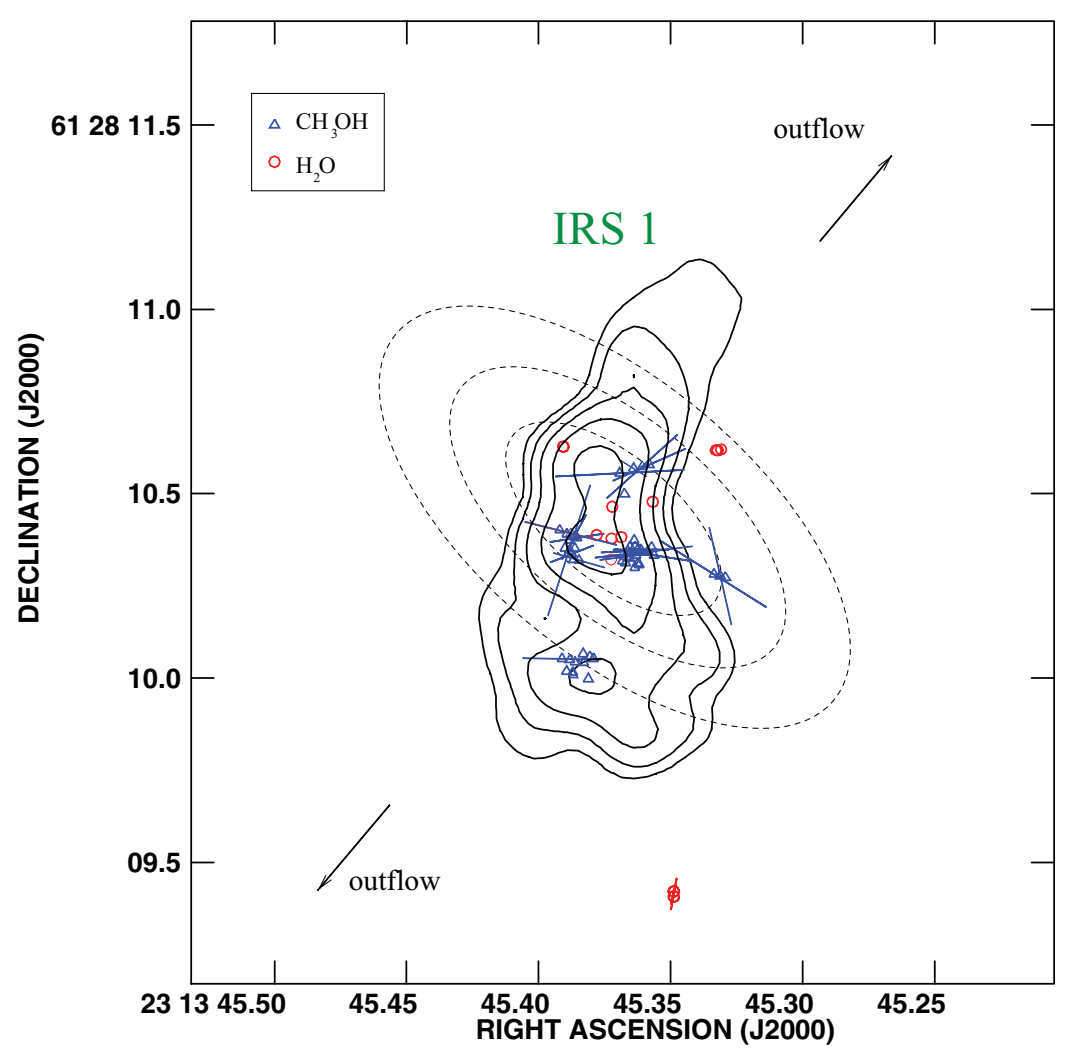

Figure 2. Modified version of Fig.1 of Surcis et al. (2011a) of the $\mathrm{CH}_{3} \mathrm{OH}$ (triangles) and $\mathrm{H}_{2} \mathrm{O}$ (circles) masers of NGC7538-IRS1. The contours are the $2 \mathrm{~cm}$ continuum emission observed with the VLA. The linear polarization vectors are also reported (60 mas correspond to a linear polarization fraction of $1 \%$ ). The dashed ellipses indicate the direction of the magnetic field.

a total molecular mass greater than $255 \mathrm{M}_{\odot}$, was also detected from $\mathrm{W} 75 \mathrm{~N}(\mathrm{~B})$ (e.g., Shepherd et al. 2003). VLA 1 was proposed to be the powering source of the outflow (e.g., Torrelles et al. 1997).

For the first time we were able to compare the orientation of the magnetic field determined from the polarized emission of two different maser species, i.e. $\mathrm{CH}_{3} \mathrm{OH}$ and $\mathrm{H}_{2} \mathrm{O}$ masers, that sample different physical characteristics. Both maser species are linearly distributed along the radio jet of VLA 1 (Fig. 1). The $\mathrm{CH}_{3} \mathrm{OH}$ masers indicate a magnetic field orientation (NE-SW) at a position angle of about $73^{\circ}$, while the field in the $\mathrm{H}_{2} \mathrm{O}$ maser region has an average position angle of $\sim 71^{\circ}$. The magnetic field derived from both maser species is thus almost aligned with the outflow, which has a position angle of $66^{\circ}$ (Hunter et al. 1994), suggesting that VLA 1 might indeed be the powering source of the outflow. We measured a magnetic field strength from the circularly polarized emission of $\mathrm{H}_{2} \mathrm{O}$ masers of about $670 \mathrm{mG}$. For more details see Surcis et al. (2009) and Surcis et al. (2011a).

\section{NGC7538-IRS1}

IRS 1 is the brightest source of the complex massive star-forming region NGC7538, which is located at a distance of $2.65 \mathrm{kpc}$ in the Perseus arm of the Galaxy (Moscadelli et al. 2009). The central star of IRS 1 has been suggested to be an O6 star of about $30 \mathrm{M}_{\odot}$ 


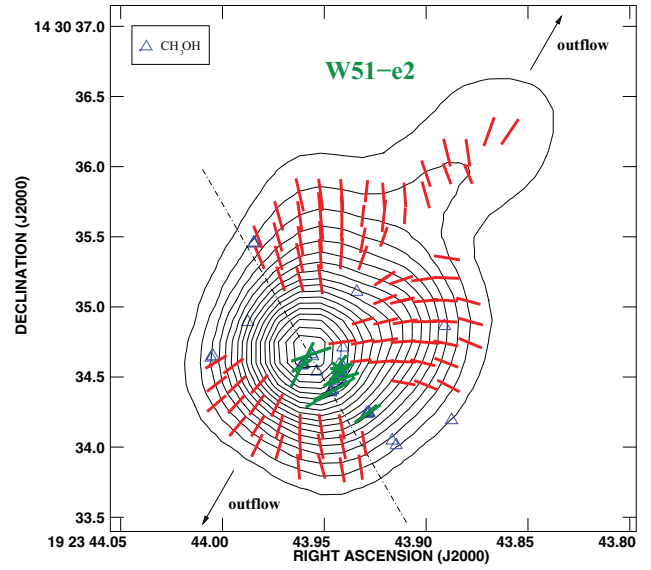

Figure 3. Modified version of Fig. 5a of Tang et al. (2009) of the dust polarization of W51-e2. The magnetic field (red segments) detected with the SMA (angular resolution $0^{\prime \prime} .7$ that corresponds to $\sim 4000 \mathrm{AU}$ ) is superimposed on the $870 \mu \mathrm{m}$ continuum contour map of W51-e2. The green segments mark the direction of the magnetic fields as derived from the $\mathrm{CH}_{3} \mathrm{OH}$ masers (angular resolution $0^{\prime \prime} .001$ corresponding to $\sim 5 \mathrm{AU}$ ). The dot-dashed black line indicates the direction of the ionized accreting flow by Keto \& Klaassen (2008).

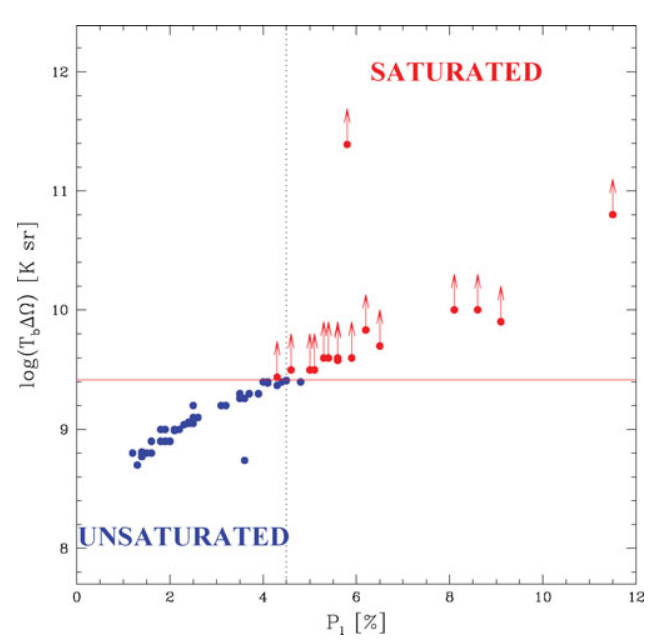

Figure 4. The emerging brightness temperatures $\left(T_{\mathrm{b}} \Delta \Omega\right)$ as function of the linear polarization fraction $\left(P_{1}\right)$ of all $\mathrm{CH}_{3} \mathrm{OH}$ masers detected so far with the EVN. The red arrows indicate that the $T_{\mathrm{b}} \Delta \Omega$ values obtained from the radiative transfer method code are lower limits. The red full line is the limit of $T_{\mathrm{b}} \Delta \Omega$ from which the $\mathrm{CH}_{3} \mathrm{OH}$ masers are considered saturated, and the dotted line shows the lower $P_{1}$ for saturated masers.

(e.g. Sandell et al. 2009). A molecular bipolar outflow $\left(\mathrm{PA}=140^{\circ}\right)$, with a velocity of about $250 \mathrm{~km} \mathrm{~s}^{-1}$ and a mass of $82.8 \mathrm{M}_{\odot}$, and a molecular torus (angular size $\sim 2$ arcsec, $\mathrm{PA}=50^{\circ}$ ) have also been detected towards IRS 1 (Qiu et al. 2011, Klaassen et al. 2009).

We detected both $\mathrm{CH}_{3} \mathrm{OH}$ and $\mathrm{H}_{2} \mathrm{O}$ masers around IRS 1 but no linearly polarized emission from the $\mathrm{H}_{2} \mathrm{O}$ masers close to the protostar was found (Fig. 2). Comparing the velocities of the $\mathrm{CH}_{3} \mathrm{OH}$ masers with the velocities of the large-scale torus we suggest that the masers are tracing the interface between the infall and the large-scale torus. The $\mathrm{H}_{2} \mathrm{O}$ masers are instead associated with the blue-shifted part of the outflow. Analysing the orientation of the linear polarization vectors of the $\mathrm{CH}_{3} \mathrm{OH}$ masers and taking into account the sign of the Zeeman-splitting measurements (positive = magnetic field points away from the observer, negative = towards the observer) we determine that the magnetic field is situated on the two surfaces of the torus with a counterclockwise direction on the top surface. For more details see Surcis et al. (2011b).

\section{W51-e2}

W51-e2 is one of the brightest molecular cores located in the eastern edge of the luminous star-forming region W51 at a distance of $5.41_{0.28}^{+0.31} \mathrm{kpc}$ (Sato et al. 2010). Keto \& Klaassen (2008) showed evidence both for infalling, or accreting, gas with a possible rotation around W51-e2 and for a bipolar outflow $\left(\mathrm{PA} \approx 150^{\circ}\right)$. The dust polarization emission at $870 \mu \mathrm{m}$ revealed a hourglass-like morphology for the inferred magnetic field (red segments in Fig. 3) near the collapsing core of W51-e2 (Tang et al. 2009). The magnetic field morphology (green segments in Fig. 3) determined from the linearly polarized emission of masers, which have been detected close to the $870 \mu \mathrm{m}$ continuum peak, is 
consistent with the hourglass morphology. This indicates that the $\mathrm{CH}_{3} \mathrm{OH}$ masers are able to probe the large-scale magnetic fields close to the protostars.

\section{Linear polarization fraction and saturation state of $\mathrm{CH}_{3} \mathrm{OH}$ masers}

We detected a total number of $213 \mathrm{CH}_{3} \mathrm{OH}$ masers towards all the 7 massive starforming regions observed with the EVN. We were able to model 72 masers by using the adapted full radiative transfer method code for $\mathrm{CH}_{3} \mathrm{OH}$ masers, which is based on the models of Nedoluha \& Watson (1992). When the code is applied to a saturated maser, it gives a lower limit for $T_{\mathrm{b}} \Delta \Omega$ and an upper limit for $\Delta V_{\mathrm{i}}$. Since the masers are unsaturated when $R / \Gamma<1$ and because the stimulated emission rate is $R \simeq A k_{\mathrm{B}} T_{\mathrm{b}} \Delta \Omega / 4 \pi h \nu$, we can estimate an upper limit for $T_{\mathrm{b}} \Delta \Omega$ below which the masers can be considered unsaturated. For $\mathrm{CH}_{3} \mathrm{OH}$ masers $\left(A=2 \times 10^{9} \mathrm{~s}^{-1}\right)$ the limit is $T_{\mathrm{b}} \Delta \Omega<2.6 \times 10^{9} \mathrm{~K}$ sr. In Fig. 4 we report $T_{\mathrm{b}} \Delta \Omega$ as function of the linear polarization fraction $\left(P_{1}\right)$. From the plot we see that all the $6.7-\mathrm{GHz} \mathrm{CH}_{3} \mathrm{OH}$ masers with $P_{1} \lesssim 4.5 \%$ are unsaturated.

\section{Conclusion}

In conclusion we have found that the magnetic field in massive star-forming regions plays a role as important as in the formation of low-mass stars. All our results are in agreement with the theoretical simulations of Banerjee \& Pudritz (2007), who demonstrated that the formation of an early outflow driven by the magnetic field is necessary in order to form the observed disc-outflow systems. From our measurements it seems that the magnetic fields are oriented along the outflow with an hourglass morphology at large-scales and when we observe closer to the protostar the magnetic field appear to be on the surfaces of a torus/disc structure from which the matter accreates onto the protostar along the magnetic field lines.

\section{References}

Banerjee, R. \& Pudritz, R. E. 2007, ApJ, 660, 479

Hunter, T. R., Taylor, G. B., Felli. M., \& Tofani, G. 1994, A\&\&A, 284, 215

Keto, E. \& Klaassen, P. 2008, ApJ, 678, L109

Klaassen, P. D., Wilson, C. D., Keto, E. R., \& Zhang, Q. 2009, ApJ, 703, 1308

McKee, C. F. \& Tan, J. C. 2003, ApJ, 585, 850

Moscadelli, L., Reid, M. J., Menten, K. M., Brunthaler, A., Zheng, X. W., Xu, Y. et al. 2009, ApJ, 693, 406

Nedoluha, G. E. \& Watson, W. D. 1992, ApJ, 384, 185

Qiu, Keping, Zhang, Qizhou, \& Menten, Karl M. 2011, ApJ, 728, 6

Rygl, K. L. J., Brunthaler, A., Sanna, A., Menten, K. M., Reid, M. J., van Langevelde, H. J., Honma, M., Torstesson, K. J. E., \& Fujisawa, K. 2012, A\&A A, arXiv1111.7023R

Sandell, G. Goss, W. M., Wright, M., \& Corder, S. 2009, ApJ, 699, L31

Sato, M., Reid, M. J., Brunthaler, A., \& Menten, K. M. 2010, ApJ, 720, 1055

Shepherd, D. S., Testi, L., \& Stark, D. P. 2003, ApJ, 584, 882

Surcis, G., Vlemmings, W. H. T., Dodson, R., \& van Langevelde, H. J. 2009, A\&̊A, 506, 757

Surcis, G., Vlemmings, Curiel, S., Hutawarakorn Kramer, B., Torrelles, J. M., \& Sarma, P. 2011a, A\&A A, 527, A48

Surcis, G., Vlemmings, W. H. T., Torres, R. M., van Langevelde, H. J., \& Hutawarakorn Kramer, B. $2011 \mathrm{~b}, A \mathscr{E} A, 533, \mathrm{~A} 47$

Tang, Y.-W., Ho, P. T. P., Koch, P. M., Girart, J. M., Lai, S.-P., \& Rao, R. 2009, ApJ, 700, 251

Torrelles, J. M., Gómez, J. F., Rodríguez, L. F., Ho, P. T. P., Curiel, S., \& Vazquez, R. 1997, ApJ, 489, 744

Vlemmings, W. H. T., Torres, R. M., \& Dodson, R. 2011, A\&A, 529, 95 